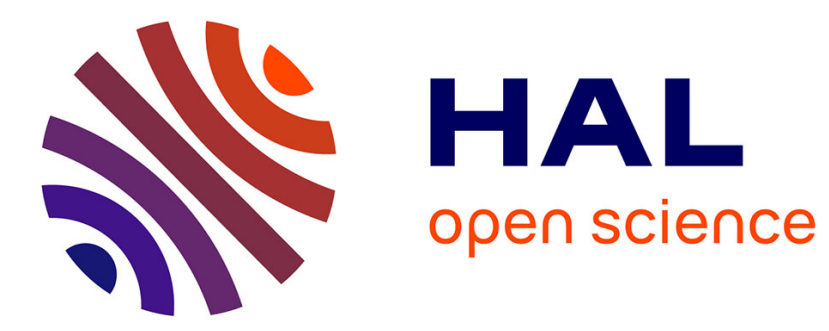

\title{
Les PTCE culture : des spécificités à ne pas négliger
} Philippe Henry

\section{To cite this version:}

Philippe Henry. Les PTCE culture : des spécificités à ne pas négliger. Revue Internationale de

l'Economie Sociale, 2017, 343, pp.88-101. 10.7202/1038782ar . hal-02307404

\section{HAL Id: hal-02307404 \\ https://hal.science/hal-02307404}

Submitted on 7 Oct 2019

HAL is a multi-disciplinary open access archive for the deposit and dissemination of scientific research documents, whether they are published or not. The documents may come from teaching and research institutions in France or abroad, or from public or private research centers.
L'archive ouverte pluridisciplinaire HAL, est destinée au dépôt et à la diffusion de documents scientifiques de niveau recherche, publiés ou non, émanant des établissements d'enseignement et de recherche français ou étrangers, des laboratoires publics ou privés. 


\section{Revue internationale de l'économie sociale}

\section{Les PTCE culture : des spécificités à ne pas négliger}

\section{Philippe Henry}

Le pôle territorial de coopération économique (PTCE)

Numéro 343, janvier 2017

URI : id.erudit.org/iderudit/1038782ar

https://doi.org/10.7202/1038782ar

Aller au sommaire du numéro

Éditeur(s)

Association Recma

ISSN 1626-1682 (imprimé)

2261-2599 (numérique)

Découvrir la revue

Citer cet article

Henry, P. (2017). Les PTCE culture: des spécificités à ne pas négliger. Revue internationale de l'économie sociale, (343), 88-101. https://

doi.org/10.7202/1038782ar

Tous droits réservés $\odot$ Recma, 2017
Résumé de l'article

Les pôles territoriaux de coopération économique culture se présentent en France comme des regroupements d'organisations à vocation coopérative, souvent de très petite taille, composées en premier lieu de producteurs et de distributeurs professionnels. Ceux-ci s'agrègent d'abord à partir d'une filière centrale d'activité artistique ou culturelle, même si une dimension de développement territorial entre aussi en jeu. Des valeurs communes de partage et de solidarité sont constamment présentes. Mais l'objectif premier porte sur une mutualisation de moyens et de compétences, de manière à ce que chaque organisation élémentaire puisse mieux faire face aux défis de survie et de développement. En cela, les PTCE culture exemplifient des questions qui n'apparaissent pas nécessairement de manière aussi exacerbée dans les regroupements de même type qui se développent dans d'autres secteurs d'activité.
Ce document est protégé par la loi sur le droit d'auteur. L'utilisation des services d'Érudit (y compris la reproduction) est assujettie à sa politique d'utilisation que vous pouvez consulter en ligne. [https://apropos.erudit.org/fr/usagers/politique-dutilisation/] 


\title{
LES PTCE CULTURE: DES SPÉCIFICITÉS À NE PAS NÉGLIGER
}

\author{
par Philippe Henry*
}

\begin{abstract}
Les pôles territoriaux de coopération économique culture se présentent en France comme des regroupements d'organisations à vocation coopérative, souvent de très petite taille, composées en premier lieu de producteurs et de distributeurs professionnels. Ceux-ci s'agrègent d'abord à partir d'une filière centrale d'activité artistique ou culturelle, même si une dimension de développement territorial entre aussi en jeu. Des valeurs communes de partage et de solidarité sont constamment présentes. Mais l'objectif premier porte sur une mutualisation de moyens et de compétences, de manière à ce que chaque organisation élémentaire puisse mieux faire face aux défis de survie et de développement. En cela, les PTCE culture exemplifient des questions qui n'apparaissent pas nécessairement de manière aussi exacerbée dans les regroupements de même type qui se développent dans d'autres secteurs d'activité.
\end{abstract}

\section{Culture PTCEs: specific features that should not be overlooked}

Regional economic cooperation clusters (pôles territoriaux de coopération économique, PTCEs) in the culture sector in France are cooperative groupings of organisations that are often very small and are primarily producers and distributors. They first group together around a core cultural activity even if there is also an element of local development. Values in common of sharing and solidarity are always in evidence. However, the main objective is the pooling of resources and competences to meet the tough challenges of survival and growth faced by every basic organisation. In this way, PTCEs in the culture sector present issues that do not necessarily appear as acutely in similar types of groupings in other sectors.

\section{Las especificidades de los PTCE cultura deben tenerse en cuenta}

Los polos territoriales de cooperación económica cultura se presentan en Francia como agrupaciones de organizaciones con vocación cooperativa, a menudo de tamaño muy pequeño, compuestas en primer lugar de productores y distribuidores profesionales. Estos últimos se agregan primeramente a partir de una actividad central artística o cultural, si bien existe también una dimensión de desarrollo territorial. Algunos valores comunes de comparición y de solidaridad están constantemente presentes. Pero el primer objetivo se centra en una mutualización de los medios y de las competencias, para que cada organización elementaría pueda hacer mejor frente a los desafíos de supervivencia y de desarrollo. Con ello, los PTCE cultura ejemplifican las cuestiones que aparecen non necesariamente de manera tan exacerbada en los agrupaciones de mismo tipo que se desarrollan en otros sectores de actividad.

* Chercheur en socioéconomie de la culture, maître de conférences HDR retraité de l'université Paris VIII-Saint-Denis. 
(1) Une forte dimension artistique et culturelle est également présente - sans être aussi fondatrice - dans d'autres PTCE, comme Initiatives et cité à Lille.
(2) Cet article présente une synthèse du compte rendu détaillé de la recherche (Henry, 2015), tout en mettant l'accent sur des éléments paraissant déterminants du point de vue de la spécificité des PTCE culture. a notion de pôles territoriaux de coopération économique (PTCE) a été officialisée aux États généraux de l'économie sociale et solidaire de juin 2011 et s'inscrit dans une réflexion plus large menée depuis 2009 (Labo de l'ESS, 2010). Un travail d'expérimentation et de caractérisation s'en est suivi dès octobre 2011, à partir d'une liste d'une vingtaine de "pôles témoins ", dont quatre centrés sur des activités artistiques et culturelles: Les Articulteurs en Pays de Redon, La Coursive Boutaric à Dijon, Culture et coopération à Saint-Etienne, la Scène de musiques actuelles de l'Ardèche ${ }^{(1)}$. Plus tard, se rajouteront Fontaine O livres et Paris Mix à Paris ou encore, signataires de la charte PTCE de septembre 2014, Le Damier à Clermont-Ferrand et Les Interactifs à Poitiers. Ainsi, sans être les plus nombreux, ces regroupements qui se reconnaissent dans le concept de PTCE - intimement lié à l'économie sociale et solidaire - n'en constituent pas moins un sous-ensemble non négligeable. Il était donc intéressant de chercher à mieux comprendre ces démarches, sur le double plan de leurs similarités et spécificités vis-à-vis des pôles ancrés dans d'autres champs d'activité.

\section{Mieux comprendre le fonctionnement des PTCE culture}

Cette approche s'est inscrite dans l'objectif de caractérisation fonctionnelle de ces nouveaux agencements coopératifs que le groupe Analyse et connaissance du Labo de l'ESS s'est prioritairement donné pour la saison 2014-2015, après sa première synthèse d'études menées en 2013 (Fraisse, 2014). Le travail a été facilité par le repérage et la description des nouvelles formes de coopération interorganisationnelle dans le domaine culturel, menés depuis plusieurs années par Opale/CRDLA - Centre de ressources culture pour le dispositif local d'accompagnement (Opale, 2011). Il a également été rendu possible grâce à l'intérêt de l'Ufisc - Union fédérale d'intervention des structures culturelles - pour les démarches de gouvernance ou de regroupement coopératifs dans ce domaine (Aufrère, 2014) et à son implication dans cette nouvelle étude ${ }^{(2)}$.

Le choix d'étude s'est porté sur quatre cas volontaires, ayant une expérience d'au moins cinq ans et se reconnaissant dans la démarche PTCE: Fontaine O livres (2007), La Coursive Boutaric (2007), Paris Mix (2008) et Culture et coopération (2010). Outre la diversité des secteurs d'activité impliqués (spectacle vivant et musiques actuelles, audiovisuel et cinéma, numérique, édition, secteur créatif), la sélection visait à éclairer les enjeux auxquels ces organisations sont confrontées une fois passée la période d'émergence et de première mise en œuvre. Une trame commune d'interrogation et d'analyse, issue des repérages 
(3) Sur les détails du processus de recherche, voir l'encadré «Précisions méthodologiques". antérieurs, a servi de socle à la recherche et de base pour des entretiens largement qualitatifs avec un responsable de la cellule d'animation-coordination des regroupements ${ }^{(3)}$. Ce panel d'observation, de fait réduit, mais déjà diversifié, a permis de dégager une série de points de repères, voire de constats récurrents, au-delà de la singularitéévidente de chacune des situations.

\section{Des éléments récurrents de positionnement}

Les quatre cas étudiés indiquent que les PTCE culture se présentent d'abord - selon une terminologie empruntée au passé - comme des agencements coopératifs de production et non de consommation (Draperi, 2012), les publics et usagers étant en effet souvent peu ou pas présents en tant que tels, comme organisations membres des regroupements ou au sein des instances décisionnaires de leurs organisations constitutives. Si le nombre de membres sous statut associatif est variable (d'un tiers pour Fontaine O livres à deux tiers pour La Coursive Boutaric en 2014), ces organisations sont très largement centrées sur des fonctions professionnalisées de production, édition, distribution ou diffusion. Les collèges " usagers " de quelques rares membres sous statut de société coopérative d'intérêt collectif (Scic) ne changent pas cette donne, confirmée par la présence plus ou moins importante selon les cas de sociétés à responsabilité limitée (SARL), de sociétés par actions simplifiée (SAS) ou même de travailleurs indépendants. Au fond, cet aspect n'a rien pour surprendre dans une économie encore massivement structurée autour d'œuvres et de démarches artistiques et culturelles, proposées à des publics par des producteurs désormais fortement professionnalisés (Henry, 2014; ICC, 2015). Ces regroupements proviennent d'initiatives associant des partenaires civils et publics. Fontaine $\mathrm{O}$ livres est issue de l'initiative de professionnels de la filière du livre et de l'édition du nord-est parisien, avec l'impulsion décisive de la Ville de Paris et le soutien de sa société d'économie mixte d'animation économique au service des territoires (Semaest). La Coursive Boutaric n'existerait pas sans l'alliance dès l'origine entre Zutique productions, association professionnelle centrée sur le développement de la scène musicale locale, et l'office public d'aménagement et de construction (Opac) de Dijon. Un partenariat entre acteurs de la filière musicale et la Ville de Paris, le conseil régional d'Ile-de-France et la direction des affaires culturelles de cette même région est à l'origine de Paris Mix. Les huit organisations civiles fondatrices de Culture et coopération avaient déjà fait leurs preuves auprès des instances publiques locales de leur capacité à mener des projets conjoints, SaintEtienne Métropole soutenant ce nouveau projet dans le cadre de l'aménagement de son quartier Manufacture. 


\section{Agrégation première et inscription territoriale}

Dans tous les cas, l'agrégation première s'est faite à partir d'une filière centrale d'activité - en l'occurrence, livre, musiques actuelles ou du monde -, même si la présence initiale ou l'extension vers d'autres activités - surtout audiovisuelles ou créatives - est également perceptible en particulier pour La Coursive Boutaric et Culture et coopération. La présence d'entreprises du numérique est également sensible et ne saurait étonner, tant la transmédiation impulsée par les nouvelles technologies est un des traits actuels majeurs d'un domaine d'activité où les relations entre pratiques artisanales et industrialisées sont en train de profondément se reconfigurer. On notera d'ailleurs qu'aucun PTCE culture ne s'est constitué à partir d'un secteur d'abord artisanal et majoritairement non marchand, comme le théâtre ou le patrimoine.

Cette polarité sur des filières particulières se double toujours d'une inscription territoriale, mais contrastée selon les cas. La prise en compte du territoire sur lequel les organisations membres du PTCE sont implantées donne ainsi lieu à des projets spécifiques, ne serait-ce que dans une optique pragmatique d'interaction avec des partenaires locaux. De même, des objectifs sociaux sont constamment présents dans chacun des regroupements, sans que l'on puisse soutenir que la dimension d'économie sociale et solidaire soit toujours revendiquée comme partie intégrante du projet initial. A ce sujet, Fontaine O livres et Paris Mix développent surtout des espaces de travail partagés, des temps d'animation et de formation, voire une fonction de pépinière d'entreprises au profit d'acteurs ou de projets qui s'initient d'abord localement. Si l'on retrouve ces éléments dans les deux autres cas, leur plus nette affirmation d'appartenance à la mouvance de l'économie sociale et solidaire les conduit aussi à développer des projets particuliers à plus fort ancrage local, comme, pour La Coursive Boutaric, la création en 2007 du Collectif Grésilles Culture « pour une programmation culturelle concertée entre les acteurs du territoire » et de la Casbah Boutaric, "fête populaire organisée au pied de l'immeuble» qui accueille le PTCE, ou encore, pour Culture et coopération, les différentes activités développées en lien avec le tissu local et à partir de 2013 par le Mixeur, «tiers-lieu et porte d'entrée sur le quartier créatif Manufacture Saint-Etienne», ou encore l'organisation depuis 2014 de la fête conviviale de quartier des Guinguettes de Manufacture.

Les quatre cas observés renvoient donc principalement à des initiatives toujours singulières, parce que situées, de regroupement progressif d'acteurs professionnels, autour d'opportunités de renforcement et de développement de l'activité de chacun, par construction ou mise en partage d'un certain nombre 
(4) Que l'on trouve déjà dans les sept principes édictés par l'Alliance coopérative internationale fondée en 1895 et qui seront repris cent ans plus tard à son congrès de 1995 (Draperi, 2012).

(5) European Alliance for Culture and the Arts, " Le pouvoir de la culture et des arts. Placer la culture au centre du projet européen ", 2016. (6) Ufisc, " L’Art est public ! Pour une politique artistique et culturelle réinventée », 2015. d'outils à visée coopérative. Ce mouvement se concrétise autour d'espaces de travail localement disponibles et, en particulier, d'un lieu commun - investi dès les premières concrétisations du projet pour les trois cas les plus anciens et seulement à partir de 2013 pour Culture et coopération -, qui facilite la relation entre les membres et une meilleure mise en œuvre des projets coopératifs. Si les PTCE culture portent bien des valeurs partagées de coopération sectorielle et territoriale, de volonté d'un développement humain plus émancipateur et solidaire, de gouvernance collégiale, ou encore d'équité de rémunération et de réserves financières non privatisables, ils apparaissent donc également comme des regroupements à visée éminemment pragmatique et issus de circonstances aussi bien contraignantes - coopérer pour survivre et se pérenniser - que capacitantes - se regrouper dans l'espoir de mieux se développer.

\section{Une relation nuancée aux principes coopératifs et à l'économie sociale et solidaire}

Même si elles n'infirment en rien l'intérêt de ce type de regroupement, les remarques précédentes renvoient néanmoins à une incertitude quant à l'application au domaine artistique - pour nous restreindre à celui-ci - des traits fondateurs du mouvement coopératif ${ }^{(4)}$, dont on trouve une résonance tant dans l'économie sociale issue des utopies associationnistes d'hier (Ferraton, 2007) que dans la mouvance actuelle de l'ESS et plus largement d'un tiers-secteur d'initiative privée mais à buts autres que lucratifs. Nous soulignerons cinq de ces traits structurants, dont la déclinaison dans les mondes de l'art ne va pas de soi.

Un premier trait affirme une finalité de service aux membres des organisations concernées ou plus largement à la collectivité tout entière, et non de profit financier pour quelques-uns. Sur ce plan, on trouve un nombre impressionnant de discours de principe mettant en exergue la dimension socialisatrice - voire universaliste - des arts et de la culture: ceux-ci « incarnent notre "vivre ensemble", font le lien entre les individus dans la société, assurent la transmission des connaissances et des valeurs » ${ }^{(5)}$, ou encore, "leurs enjeux relèvent des droits humains, du bien commun et de "l'intérêt général", dans une vision qui transcende la somme des intérêts particuliers, les corporatismes et les replis sur soi ${ }^{\left({ }^{(6)}\right.}$. Fort bien. Mais qu'en est-il, dans les faits, de la tension entre ces positions humanistes et la singularisation méritocratique et élitaire du fonctionnement contemporain des mondes de l'art (Heinich, 2005)? Et pour les organisations membres des PTCE culture, qu'en est-il concrètement de leur proximité ou de leur distance avec les critères d'aide aux personnes en situation de fragilité ou de lutte contre les exclusions et les inégalités, d'ouverture à tous, de fonctionnement démocratique, de transparence 
(7) Haut Conseil à la vie associative, « Rapport sur la notion d'intérêt général fondant l'intervention des associations ", 2016. financière et de compte rendu d'activité - outre ceux de gestion désintéressée et de non-lucrativité -, que le Haut Conseil à la vie associative propose comme balises pour mieux qualifier les organismes d'intérêt général ${ }^{(7)}$ ?

La revendication d'une autonomie de gestion et d'une indépendance de décision des organisations vis-à-vis de pouvoirs externes - qu'ils soient publics ou privés - est un second trait du mouvement coopératif. Si on a là un discours en phase avec une bonne partie du domaine artistique, reste que l'on sait que ces dimensions sont très relatives et doivent toujours plus s'appréhender en tant que revendication d'une liberté réelle de fonctionnement dans le cadre d'une interdépendance de fait renforcée entre instances et organisations.

Dans le processus interne de décision, place doit être faite à une véritable dimension démocratique, quel que soit l'apport financier ou de compétence de chacun à l'organisation, même si elle n'aboutit pas nécessairement à la seule règle où une personne dispose d'une voix délibérative. La concrétisation de ce principe dans les organisations du domaine artistique, y compris dans sa part la plus " sociale et solidaire », est sans doute une des plus problématiques.

En termes de répartition autant des revenus que d'éventuels excédents, primauté est donnée à la personne coopérante et à l'objet social de l'organisation et non à la rémunération privée du capital. Si une bonne partie du domaine artistique adhère idéalement à ce principe, la répartition très inégale des ressources et des notoriétés - ici encore, tiers-secteur compris - est un aspect central jamais facile à aborder, comme le montre la question du régime de rémunération socialisée qui serait le plus " juste " pour les artistes et techniciens du spectacle ou ceux de la littérature et des arts plastiques, en situation d'activité constante et de travail rémunéré discontinu (Bureau et Corsani, 2012).

Enfin, on mentionnera le principe de l'adhésion libre de chacun à l'organisation coopérative, un retrait personnel étant toujours possible, l'engagement signifiant a contrario une co-obligation effective entre les membres des organisations concernées, dont fait partie la formation des plus jeunes. Une fois encore, pour le domaine artistique, c'est la mise en œuvre concrète et dans la durée de ce principe qui serait à regarder de plus près, même s’il semble a priori le moins problématique ou conflictuel.

Aux éléments précédents, on rajoutera une remarque décisive pour aujourd'hui. L'extension et l'intensification d'une économie plus coopérative et solidaire tiennent également à la capacité des expériences en cours à convaincre une part plus importante et agissante de nos concitoyens et des pouvoirs publics non seulement de l'intérêt, mais aussi de la nécessité renforcée d'aider au développement d'une telle dynamique, d'abord portée par la société civile. Rien d'évident quand on voit le primat actuel donné 
aux principes d'individualisme, de concurrence par le marché et de compétitivité interentreprise, que ce soit au niveau des cultures dominantes ou à celui plus directement politique des Etats nationaux et de l'Union européenne.

Tout compte fait, les PTCE culture pourraient aisément rentrer dans la définition formalisée - et assez extensive - donnée par la loi de juillet 2014 des organisations et entreprises relevant de l'économie sociale et solidaire. Mais dans leur fonctionnement concret, elles oscillent probablement bien plus entre, d'une part, une référence idéologiquement forte au mouvement coopératif tel qu'on vient d'en rappeler quelques principes et, d'autre part, une appartenance de fait à l'entrepreneuriat social (Barthélémy et Slitine, 2011) qui concerne, selon le Mouvement des entrepreneurs sociaux (Mouves), les «entreprises à finalité sociale, sociétale ou environnementale et à lucrativitélimitée [qui] cherchent à associer leurs parties prenantes à leurgouvernance». Cette double question de la lucrativité limitée et de la gouvernance partagée ne fait pas débat en termes de fonctionnement interne des PTCE culture - et notamment de leur cellule d'animation-coordination. Mais elle n'est pas forcément au cœur du projet de chacune de leurs organisations membres, en premier lieu confrontées à l'incertitude de la valorisation sociale et économique de leur activité.

La compréhension des PTCE culture pourrait également gagner à se référer à la théorie renouvelée des communs, considérés comme "ensembles de ressources collectivement gouvernées, au moyen d'une structure de gouvernance assurant une distribution des droits entre les partenaires participant au commun et visant à l'exploitation ordonnée de la ressource, permettant sa reproduction sur le long terme » (Coriat, 2015). En tant que mise en œuvre d'un partage et d'un développement collectif de ressources matérielles et immatérielles, chaque PTCE culture répond à cette définition. A ceci près qu'il faudrait regarder de plus près comment se joue, dans chaque cas, l'équilibre entre la conservation de droits - y compris exclusifs - d'exploitation et d'administration sur certaines ressources produites collectivement, et l'ouverture de leur accès et de leur utilisation à un plus grand nombre. On retombe ici sur la question déjà évoquée de la nature et de l'extensivité de ce "plus grand nombre ", mais aussi sur celle des modalités organisationnelles concrètes par lesquelles cette distribution différenciée des droits peut effectivement se réaliser.

(8) Le décret $n^{\circ}$ 2008-1354 du 18 décembre 2008 définit les microentreprises comme celles occupant moins de dix personnes et dont le chiffre d'affaires par an ou le bilan est inférieur à 2 millions d'euros.

\section{Une relation variable au principe d'une gouvernance partagée}

Une autre dimension marquante et qui dépasse les quelques cas de PTCE culture étudiés tient au fait qu'ils relèvent massivement du regroupement de microentreprises ${ }^{(8)}$, le nombre de celles ne comportant qu'une personne initiatrice ou guère plus 
d'un salarié pouvant être non négligeable. Si des entreprises de plus fort potentiel sont souvent à l'origine du regroupement, les plus grosses organisations membres - la plupart du temps relevant d'un statut d'établissement public ou d'une délégation de service public pour la gestion d'un équipement important - sont rarement très actives ou centrales. Apparaît d'ailleurs souvent la question de l'optimum d'activité et de développement au-delà duquel apparaissent - pour chaque organisation membre du regroupement ou pour celui-ci dans son ensemble - des phénomènes de saturation de l'agenda des membres ou de perte de pertinence et d'efficacité du projet coopératif. La dimension, elle aussi décisive, de proximité relationnelle et fonctionnelle entre les acteurs peut en effet assez vite se déconstruire au-delà d'une certaine étendue et complexité organisationnelles.

Dans ces conditions, de nombreuses organisations membres souhaitent réellement coopérer sur des projets particuliers ou au travers d'activités communes ou partagées, sans pour autant vouloir par trop s'impliquer dans la gouvernance et l'animation d'ensemble du regroupement. Cette observation expliquerait une partie des aléas rencontrés pour initier, maintenir et développer sur la durée une implication réelle de la totalité des membres dans la gouvernance générale du regroupement, alors même que des coopérations sur des projets particuliers ou des aspects plus délimités continuent à s'inventer et à être à l'œuvre. Les statuts de Fontaine $\mathrm{O}$ livres distinguent ainsi des membres actifs avec droit de vote aux assemblées générales et des membres utilisateurs ne disposant pas de ce droit. Sur la quarantaine de membres de Paris Mix, douze - dont beaucoup de fondateurs - constituent le conseil d'administration se réunissant chaque trimestre et forment le noyau central du PTCE. Une organisation en groupes de travail thématiques ne rassemblant régulièrement que certains des membres est également souvent perceptible, comme dans le cas de Culture et coopération ou Paris Mix. Si le nombre de membres officiels des regroupements est très variable, pouvant aller jusqu'à plusieurs dizaines, on maintiendra donc l'hypothèse que chacun des PTCE culture est centralement porté par plus ou moins une dizaine de membres, au titre de ceux qui se reconnaissent et s'investissent fortement et sur la durée dans l'organisation du regroupement, le développement des activités partagées ou sa cellule d'animation-coordination. Souvent d'ailleurs, l'implication réelle dans le regroupement est principalement le fait d'une personne de ces organisations, qui assume aussi en général une fonction de direction dans chacune d'entre elles. Ces constats rejoignent ceux que l'on peut faire sur des PTCE relevant d'autres secteurs d'activité, où l'on repère l'existence de "cercles d'appartenance imbriqués » et une distinction souvent peu nette entre membres et non-membres des regroupements 
selon l'implication des organisations élémentaires dans les projets concrets de coopération, eux-mêmes à géométrie variable tant dans leur objet que dans leur durée (Fraisse, 2015).

\section{Interdépendance collaborative et singularisation}

On aurait tort d'en tirer la conclusion d'une faiblesse des PTCE culture sur ce point. Mieux vaut surtout tenir compte de leurs spécificités socioéconomiques qui, pour une part, ne font qu'exacerber des dynamiques repérables dans d'autres secteurs d'activité. A nouveau, l'exemple des mondes de l'art illustre un fonctionnement où priment les interactions réticulaires. L’interdépendance collaborative entre acteurs différenciés y apparaît donc bien comme une dynamique essentielle (Becker, 1982) et plaiderait pour des formes intensifiées et renouvelées de coopération interorganisationnelle. Sauf que cette dynamique se heurte à une redoutable concurrence par les qualités, dans la mesure où les dispositifs de valorisation sont toujours sélectionnants, classants et donc hiérarchisants. Loin d'être un phénomène périphérique, cette situation renvoie à la structure même de l'économie artistique - et plus largement culturelle -, toujours profondément enchâssée dans une réalité sociale spécifique (Greffe, 2010). Cette économie se caractérise en effet par la production et l'appropriation de biens singuliers - ou pour le moins très fortement différenciés - et donc peu (ou pas) substituables les uns aux autres. La pertinence-utilité de ces biens ou services est donc a priori très incertaine, et ne sera qualifiée qu'au travers d'un processus social de valorisation, rarement simple et court, et dans lequel l'intersubjectivité tient une place déterminante (Karpik, 2007). Cette situation d'incertitude constante et structurelle quant au devenir des propositions culturelles se répercute d'autant plus sur les organisations qui les portent que celles-ci sont de petite taille, les particularités d'intégration au marché de ces organisations s'en trouvant encore renforcées (Mallard, 2011). Chacune se trouve alors prise dans un jeu permanent, d'un côté, de différenciation et de singularisation de ses propositions et, de l'autre, d'interdépendance renforcée avec les différents acteurs de sa filière d'activité, qu'ils soient petits ou gros, situés en amont ou en aval de ces filières.

Ce sont finalement ces tensions irréductibles que l'on retrouve au sein des PTCE culture, d'autant qu'ils peuvent également regrouper des organisations ne relevant pas de la même filière culturelle. En tout cas, la question se trouve constamment posée de l'équilibre à trouver entre la pérennité nécessaire de l'identité de chaque organisation et le développement non moins nécessaire d'une identité propre du regroupement coopératif. 
La réticence à plus s'impliquer dans la gouvernance d'ensemble du PTCE peut alors aussi renvoyer à l'inquiétude - fréquente chez les partenaires les plus petits - de la perte de leur identité propre au sein du regroupement. Celle-ci constitue en effet un élément majeur dans un milieu culturel où l'horizon d'activité de chaque organisation est, de plus, souvent de court terme et très incertain.

Ces différents éléments renforcent encore le rôle essentiel de la fonction d'animation-coordination des PTCE culture. Dans les cas observés, celle-ci s'appuie sur une structure juridique - association type loi de 1901, divers projets de passage en Scic étant étudiés mais à ce jour non aboutis. Quoi qu'il en soit, c'est d'abord l'articulation d'agencements formels et informels qui apparaît déterminante. Au-delà des échanges interpersonnels toujours fondamentaux, la réalité quotidienne des processus de régulation et de prise de décision tient ainsi beaucoup aux divers groupes de travail et séminaires plus ou moins réguliers que la cellule d'animation-coordination saura initier ou soutenir dans la durée. On retrouve ici des déclinaisons particulières d'une organisation matricielle, où la dimension verticalisée de la gouvernance - un bas et un haut en interaction réglée - ne tient surtout que par l'existence d'une dimension horizontalisée construite autour de groupes de projet ou de comités de pilotage, où s'impliquent plus fortement les membres concernés par des sujets particuliers. Au bout du compte, on est face à des dynamiques régulatrices qui se gardent de dispositifs trop formalisés, tout en déployant une gamme de formats délibératifs, de boucles d'échange, de niveaux de prise de décision assez conforme à la complexité sans cesse mouvante du regroupement et de l'activité de ses membres. Dans de telles configurations organisationnelles, la question des personnes en capacité de faciliter l'articulation globale d'enjeux diversifiés et de modes mouvants de coopération pose en tout cas celle de la place et de la forme d'un leadership attentif au maintien d'une cohésion dynamique suffisante entre acteurs, toujours par ailleurs préoccupés par leur propre devenir.

\section{L'importance des visées pragmatiques et opératoires et la difficulté d'un modèle économique autonome et pérenne}

$\mathrm{Au}$ vu de ce qu'on vient de souligner, il n'est pas surprenant de constater la place accordée dans les PTCE culture aux objectifs concrets d'adaptation socioéconomique par épaulement réciproque des membres en vue d'amplifier leur propre activité. Sur ce plan, la proximité avec les PTCE d'autres secteurs d'activité est perceptible. Apparaissent ainsi premiers les objectifs tels que: une optimisation des ressources disponibles, par mutualisation 
significative de moyens matériels, de compétences ou de financements; un partage et une veille élargis d'informations sur les opportunités et les évolutions du secteur d'activité ou du territoire; la recherche d'une taille critique pour mener collectivement des projets de plus grande ampleur; mais aussi, la création de nouveaux services, le développement de coopérations visant à la structuration territoriale d'une filière ou d'un secteur d'activité; ou encore le renforcement d'une capacité collective d'ingénierie territoriale et de développement local. Au-delà d'indispensables valeurs et ressorts idéologiques partagés qui constituent un élément premier dans les regroupements coopératifs arrivant à perdurer, comme dans le spectacle vivant (Sinapi et Juno-Delgado, 2015), la capacité de survie et de développement des PTCE culture tient à la réalisation au moins partielle de tels objectifs.

Ces derniers se concrétiseront par des mutualisations et propositions de service portant d'abord sur les membres du regroupement, même si la volonté de proposer dès que possible des services à des acteurs externes est aussi sensible. Tout un gradient d'activités va ainsi apparaître, que chaque PTCE déclinera à sa manière: mutualisation partielle de compétences et de ressources (en particulier matérielles et immatérielles, dont le partage de locaux et d'informations); mutualisation déjà plus intensive de fonctions supports (comme des outils communs de communication, des achats groupés, la gestion d'emplois partagés ou la formation); coopération économique sur des projets particuliers (depuis des coopérations bilatérales jusqu'au développement de projets communs inédits, en passant par une offre collective de services à des acteurs externes au regroupement). $\mathrm{Au}$ fil du temps, un véritable "surplus coopératif » apparaîtra ainsi, par différence avec ce que chaque organisation aurait pu réaliser isolément (Deniau, 2014). Compte tenu de ce que nous avons dit de l'environnement socioéconomique des PTCE culture, le partage d'une visée stratégique et d'une prospective commune entre leurs membres et du point de vue d'un territoire ou d'un secteur d'activité donné apparaît plus difficile à concevoir - ou pour le moins ne peut rester que partielle -, ce qui ne retire rien à l'intérêt des autres strates de coopération.

Les cas étudiés soulignent aussi la très grande importance des moments d'échange informel et convivial entre les membres, que permettent le lieu commun ou les temps réguliers de rencontre qui sont organisés. Plus particulièrement et au-delà des très nombreux projets élémentaires partagés ayant pu être menés entre les membres, on repère aussi des projets structurants et diversifiés tels que: un portail des compétences disponibles (La Coursive Boutaric), une centrale des marchés solidaires pour l'accès aux marchés publics (Culture et coopération); des espaces ou événements de promotion 
(9) Preuve s'il en était besoin de la fragilité structurelle de ces démarches, Paris Mix a cessé son activité en novembre 2015 et Culture et coopération en décembre 2016. liés aux activités des membres (Fontaine O livres, Paris Mix), mais aussi des propositions d'expertise ou de conseil en ingénierie culturelle (Fontaine O livres, La Coursive Boutaric); des espaces de travail partagés, mais aussi un incubateur de projets créatifs (Culture et coopération) ou une pépinière d'entreprises (Fontaine O livres); deux groupements d'employeurs (Paris Mix-Fontaine O livres, Culture et coopération).

\section{Le point nodal des outils de solidarité économique}

Quant aux modèles économiques de la fonction d'animationcoordination des PTCE culture, ils reposent essentiellement sur les contributions volontaires de leurs différents membres et sur des financements publics - nationaux ou plus territoriaux. La contribution régulière des membres pour couvrir le coût des moyens mutualisés repose moins sur des cotisations annuelles très peu élevées que sur la participation aux charges du lieu commun et, surtout, sur les transferts financiers liés aux emplois mutualisés qui constituent la modalité principale de coopération financière. Les contributions en compétences fournies entre membres représentent la seconde ressource principale, mais ne font généralement pas l'objet d'une comptabilisation, ne serait-ce qu'en termes de temps passé. Les recettes propres liées aux prestations de service proposées à des acteurs externes au regroupement ne sont pas négligeables parfois, mais restent, dans l'ensemble, encore très limitées (de $7 \%$ pour Culture et coopération à $37 \%$ pour Fontaine $\mathrm{O}$ livres). L'équilibre financier de la fonction d'animation-coordination repose donc encore très majoritairement sur des financements publics. Comme ceux-ci correspondent pour beaucoup à des dispositifs limités dans le temps, cela fait effectivement peser sur chacun des regroupements une réelle incertitude quant à leur avenir à moyen et long terme.

De fait, la double question de la nécessité et de la capacité d'outils de solidarité financière entre les membres des PTCE culture est un point nodal. Une fois de plus, l'impératif de réinvention constante de l'activité des membres, leur incertitude structurelle quant au moyen et long terme au sein d'un contexte fortement concurrentiel et mouvant, la fragilité économique induite de chacun, tous ces éléments rendent très délicate la concrétisation de ce genre de perspective ${ }^{(9)}$. Quelle qu'en soit la difficulté, une plus nette mise en commun des ressources économiques et financières de chacun des membres du regroupement apparaît néanmoins comme une des urgences structurelles dans l'objectif d'un développement et d'une pérennisation plus assurés des PTCE culture, ce point se retrouvant aussi clairement pour ceux relevant d'autres domaines d'activité. 


\section{Conclusion}

Pour conclure, on soulignera à nouveau combien les PTCE à forte centration artistique et culturelle doivent être appréhendés au prisme de la socioéconomie propre à leur domaine d'activité. Cet aspect a été un fil rouge des lignes précédentes. A minima, il impliquerait de compléter et d'affiner la représentation idéelle des ces regroupements, telle que promue tant par les militants de l'économie sociale et solidaire que par les pouvoirs publics. Symétriquement, ces PTCE fournissent des indications précieuses sur les opportunités et les modalités de fonctionnement de ce type de regroupement. Leur subtilité et complexité managériales apparaissent alors autant comme sources d'inspiration et d'expérimentation que comme rappels des contingences et des difficultés à prendre explicitement en compte par tous ceux qui rêvent d'un monde plus coopératif et solidaire, plus que jamais urgent à promouvoir dans les temps troublés qui sont les nôtres.

\section{Précisions méthodologiques}

Cette approche des PTCE culture a été menée entre octobre 2014 et mai 2015. Elle s'appuie sur une déclinaison au domaine culturel d'une trame analytique d'observation, issue des constats synthétiques réalisés en 2013-2014 par le groupe Analyse et connaissance du Labo de l'ESS sur un ensemble de PTCE de divers secteurs d'activité. Cette trame s'organise autour de cinq dimensions: morphologie du regroupement; objectifs et besoins identifiés; activités de coopération mises en œuvre; critères d'appartenance au regroupement et modalités de gouvernance; économie du projet coopératif.

Sur cette base, l'enquête proprement dite a d'abord reposé sur la passation d'un questionnaire largement qualitatif en face-à-face entre l'enquêteur et un responsable de la cellule d'animation-coordination des regroupements. Après un premier entretien de l'ordre de trois heures et une récolte complémentaire de documents, une mise en forme écrite des caractéristiques principales du regroupement sur les cinq dimensions d'analyse a donné lieu à un second entretien de confirmation et d'approfondissement. Les moyens de recherche disponibles n'ont pas permis une interrogation de plusieurs personnes clés du regroupement coopératif. Le processus de recherche a en effet essentiellement reposé sur le temps qu'ont pu dégager Alban Cogrel sur son poste de chargé de mission sur les questions de structuration territoriale et socioéconomique à l'Ufisc (d'abord pour le travail d'enquête sur les cas) et Philippe Henry sur ses activités de retraité de l'université (pour le cadrage méthodologique et le travail d'interprétation). Dans la mesure où une partie de la trame analytique est constituée par une demande de pondération relative de propositions élémentaires - une note à donner de 4 à 0 selon l'importance accordée à tel ou tel item -, les résultats présentés reposent sur la perception de l'histoire et du mode de fonctionnement du PTCE par le responsable interrogé. Ce point signalé, il ne constitue plus forcément un biais d'analyse. Il apparaît surtout comme une approche située, qui pourrait tout à fait être reprise et travaillée de manière plus contrastée et collective au sein de chaque regroupement étudié.

La densité des informations recueillies, sur chacune des cinq dimensions d'exploration, a pu être variable selon les cas. Les données objectives et quantifiées, aidant à mieux appréhender la morphologie de ce qui fait le cœur du regroupement, ont été les plus difficiles à constituer. 


\section{BIBLIOGRAPHIE}

Aufrère L., 2014, Enjeux et perspectives $d u$ renouvellement de la gouvernance collective dans les associations artistiques et culturelles, Crida/ Opale/Ufisc.

Barthélémy A. et Slitine R., 2011, Entrepreneuriat social: innover au service de l'intérêt général, Paris, Vuibert.

Becker H. S., 1982, Les mondes de l'art, traduit par Jeanne Bouniort (1988), Paris, Flammarion.

Bureau M.-C. et Corsani A. (dir.) , 2012, Un salariat au-delà du salariat ?, Nancy, PU de Nancy-Editions universitaires de Lorraine.

Coriat B. (dir.), 2015, Le retour des communs : la crise de l'idéologie propriétaire, Paris, Les Liens qui libèrent.

Deniau M., 2014, Etude exploratoire sur les nouvelles pratiques de mutualisation ou de coopération inter-organisationnelles dans le secteur culturel, Paris, DEPS-Ministère de la Culture et de la Communication.

Draperi J.-F., 2012, La république coopérative, Bruxelles, éditions Larcier.

Ferraton C., 2007, Associations et coopératives. Une autre histoire économique, Toulouse, éditions Erès.

Fraisse L., 2015, Caractériser les pôles territoriaux de coopération économiques : premiers résultats, Labo de l'ESS/Institut CDC pour la recherche.

Fraisse L. (coord.), 2014, « Pôles territoriaux de coopération économiques : synthèse des études 2013 ", groupe Analyse et connaissance, Le Labo de l'ESS.

Greffe X., 2010/1, «Introduction : l'économie de la culture est-elle particulière ? ", Revue d'économie politique, vol.120, pp.1-34.
Heinich N., 2005, L'élite artiste : excellence et singularité en régime démocratique, Paris, Gallimard.

Henry P., 2015, Pôles territoriaux de coopération économiqueculture: des regroupements pragmatiques dans des secteurs d'activité de grande incertitude, disponible sur Opale.assoc.fr. Henry P., 2014, Un nouveau référentiel pour la culture? Pour une économie coopérative de la diversité culturelle, Toulouse, éditions de l'Attribut.

ICC-Institut de coopération pour la culture, 2015, Investir en urgence dans des modèles économiques de la création artistique plus coopératifs et solidaires.

Karpik L., 2007, L'économie des singularités, Paris, Gallimard.

Labo de l'ESS, 2010, Pour une autre économie : 60 propositions pour changer de cap, hors-série poche $\mathrm{n}^{\circ} 46$ bis, Paris, Alternatives économiques.

Mallard A., 2011, Petit dans le marché : une sociologie de la très petite entreprise, Paris, Presses des Mines.

Opale, 2011, "Les coopérations culturelles : fiche expérience »-Le cluster de Saint-Etienne "Culture \& Coopération"; Le cluster "Les Articulteurs "; Pôle Max Jacob, Quimper.

Sinapi C. et Juno-Delgado E., 2015, « Motivations for Establishing Cooperative Companies in the Performing Arts : A European Perspective", in Kauhanen A. (dir.), Advances in the Economic Analysis of Participatory and Labor-Managed Firms, Emerald Group Publishing Limited, vol. 16, pp. 63-107. 